\title{
Sparse Approximation of Singularity Functions
}

\author{
Pál-Andrej Nitsche
}

\begin{abstract}
We are concerned with the sparse approximation of functions on the $d$ dimensional unit cube $[0,1]^{d}$, which contain powers of distance functions to lowerdimensional $k$-faces (corners, edges, etc.). These functions arise, e.g., from corners, edges, etc., of domains in solutions to elliptic PDEs. Usually, they deteriorate the rate of convergence of numerical algorithms to approximate these solutions.

We show that functions of this type can be approximated with respect to the $H^{1}$ norm by sparse grid wavelet spaces $V_{L}, \operatorname{dim}\left(V_{L}\right)=N_{L}$, of biorthogonal spline wavelets of degree $p$ essentially at the rate $p$ :

$$
\left\|u-P_{L} u\right\|_{H^{1}\left([0,1]^{d}\right)} \leq C N_{L}^{-p}\left(\log _{2} N_{L}\right)^{s}\|u\|, \quad s=s(p, d),
$$
\end{abstract}

where $\|\cdot\|$ is a weighted Sobolev norm and $P_{L} u \in V_{L}$.

\section{Introduction}

The efficient numerical approximation of functions of several variables is required in numerous applications: In the numerical solution of partial differential equations (PDEs) in dimension 3 or higher, in data mining, in numerical integration over high-dimensional domains, to name but a few.

Tensor products of piecewise polynomial functions of degree $p$ on a uniform mesh of width $h>0$ require $\mathcal{O}\left(h^{-d}\right)$ degrees of freedom and achieve an asymptotic convergence rate in the $H^{1}$ norm of $\mathcal{O}\left(h^{p}\right)$ if the $(p+1)$ st derivatives of the function to be approximated are square-integrable.

The growth as $h \rightarrow 0$ of the number of degrees of freedom in tensor product interpolants is excessive if $d$ is large. So-called sparse interpolants - which require only $\mathcal{O}\left(h^{-1}|\log h|^{d-1}\right)$ degrees of freedom rather than $O\left(h^{-d}\right)$ - have been proposed and successfully used in applications (see, e.g., [8], [16], [26]). There it was shown that despite their reduced number of degrees of freedom, sparse tensor product interpolants can achieve an $H^{1}$ convergence rate of $\mathcal{O}\left(h^{p}|\log h|^{d-1}\right)$, i.e., up to logarithmic terms the rate of the full tensor product approximation is preserved. This is possible, however, only under very strong assumptions on the regularity of the function to be approximated:

Date received: September 19, 2002. Date revised: November 4, 2003. Date accepted: November 28, 2003. Communicated by Wolfgang Dahmen. Online publication: March 25, 2004.

AMS classification: $65 \mathrm{~N} 12,41 \mathrm{~A} 25,65 \mathrm{~T} 60$.

Key words and phrases: Sparse grids, Elliptic singularities, Approximation of singularities, Bioorthogonal wavelets. 
Certain derivatives up to order $d(p+1)$ must be square-integrable in order to realize the convergence rate $\mathcal{O}\left(h^{p}|\log h|^{d-1}\right)$ in $H^{1}$.

In particular, in the numerical treatment of PDEs the solutions are not that regular, for example, due to corner singularities in elliptic problems. The potential gain in using sparse tensor product approximation is strongly limited in this case-the reduction in the convergence rate essentially offsets the reduction in degrees of freedom in the interpolant.

Singularities in solutions of elliptic problems contain as essential parts powers of distance functions to the singular support of the domain (corner, edges, etc.). For instance, for polygonal domains $\Omega$ in two dimensions, there holds a decomposition of the weak solution $u$ to a uniformly elliptic boundary value problem into

$$
u=u_{\mathrm{reg}}+u_{\mathrm{sing}},
$$

with smooth $u_{\text {reg }}$ and a finite sum

$$
u_{\text {sing }}=\sum^{<\infty} \operatorname{Re}\left(r_{i}^{\lambda_{i}}\left(\log r_{i}\right)^{\mu_{i}} \Phi_{i}\left(\varphi_{i}\right) \eta_{i}(x)\right),
$$

(in local polar coordinates $\left(r_{i}, \varphi_{i}\right)$ associated to the corners (here not indexed)) with smooth cut-off functions $\eta_{i}$, smooth functions $\Phi_{i}$ of the angle coordinates $\varphi_{i}$, and parameters $\lambda_{i} \in \mathbf{C}, \mu_{i} \in \mathbf{N}_{0}$, see, e.g., [5], [6], [7]. For Dirichlet-Dirichlet corners, one knows $\operatorname{Re} \lambda_{i}>\frac{1}{2}$, and for Dirichlet-Neumann corners, $\operatorname{Re} \lambda_{i}>\frac{1}{4}$.

In higher dimensions, decomposition theorems are not that easy to write down, but estimates on the growth (of the function and its derivatives) toward the singular support of the domain are known, see, e.g., [19].

The rate of approximation of singular functions determines the convergence rate of Finite Element Methods for elliptic problems. The dimension $N$ of FE spaces of continuous, piecewise polynomials of degree $p$ on a quasi-uniform mesh of mesh width $h$ is $N=\mathcal{O}\left(h^{-d}\right)$. For solutions $u \in H^{s+1}(\Omega)$, the $H^{1}$ convergence rate of the best approximation is $\mathcal{O}\left(N^{-\min \{p, s\} / d}\right)$. In dimension $d=2$, singular terms of the above type belong to $H^{s+1}(\Omega)$ with $s<\operatorname{Re}(\lambda)$.

To overcome the reduced convergence rate, the nonuniform distribution of degrees of freedom by local mesh refinement has been proposed. This can be done a priori, i.e., by grading the mesh toward the singular support of (1), or a posteriori, by adaptive mesh refinement and nonlinear approximation.

In dimension 2, these techniques allow us to recover the maximal asymptotic rate $\mathcal{O}\left(N^{-p / 2}\right)$, see, e.g., [3], [20] for recent results and, in particular, [9], where it has been proven that the rate $\mathcal{O}\left(N^{-b}\right)$ is achievable by adaptive (nonlinear) wavelet approximation whenever such a rate is possible by so-called best $N$-term approximation (for related numerical results, see also [2]). The result in [9] is, in particular, not restricted to functions of the special type (1), but applies to all functions which belong to a scale of suitable Besov spaces (see [13] for details).

The result [9] applies to certain FEM bases with "isotropic" support, or with "shape regular" elements in FE terminology. In polyhedra $\Omega$ in dimension $d=3$, the appearance of edge singularities with anisotropic regularity does not allow us to achieve the rate $\mathcal{O}\left(N^{-p / d}\right)$ with local mesh refinements, as long as the basis is isotropic. However, this rate can be achieved with suitable anisotropic mesh refinements (see, e.g., [1]). 
In the present paper, we therefore analyze the approximation of singular functions built from powers of distance functions to corners, edges, etc., in anisotropically refined sparse tensor product spaces.

We prove that anisotropic sparse tensor product spaces (of piecewise polynomials of degree $p$ ) allow us to approximate corner and edge singularities in dimension 3 (defined on $\left.[0,1]^{d}\right)$ at a rate

$$
\mathcal{O}\left(N^{-p}\left(\log _{2} N\right)^{s}\right), \quad s=2 p+\frac{3}{2},
$$

in the $H^{1}$ norm, where $N$ is the number of degrees of freedom in the sparse, anisotropic tensor product. In particular, (2) shows that the reduction in the convergence rate, due to the higher dimension and low Sobolev regularity, can be eliminated (up to logarithmic terms).

Our proof is not restricted to dimension 3, but applies to any dimension $d>1$. In particular, and at first sight somewhat surprisingly, even in dimension 2, the use of sparse tensor products of wavelet bases with local refinement for the approximation of "isotropic" corner singularities (1) gives an improvement over the "optimal" rate $\mathcal{O}\left(N^{-p / 2}\right)$ in the $H^{1}$ norm: We obtain that, for each polynomial degree $p \geq 1$, there is a sequence of sparse spline wavelet approximants of degree $p$ which converges at the rate $\mathcal{O}\left(N^{-p}\left(\log _{2} N\right)^{p+1}\right)$, as opposed to the rate $\mathcal{O}\left(N^{-p / 2}\right)$ obtained from nonlinear, adaptive approximation in the "isotropic" setting (i.e., support of wavelet bases and Besov spaces used to measure the regularity of (1) are isotropic).

Let us point out that our result immediately yields upper bounds on nonlinear and adaptive approximation schemes in anisotropic wavelet bases for the elliptic singularities, which are superior to those of [9] and [3] in the isotropic setting. These connections will be elaborated elsewhere.

The remainder of this paper is organized as follows: In Section 2 we introduce the wavelets we use and prove the one-dimensional results; the key result is Lemma 3. In Section 3 we consider the two-dimensional case and give a detailed proof of the twodimensional consistency estimate using an abstract tensor product argument. In Section 4 we extend the two-dimensional results to $d$ dimensions by iterating the arguments of Section 3. The main theorems are Theorems 4 and 5.

Concerning the application to singular functions arising from corners of polygonal domains or from changes in the type of boundary conditions, Theorem 3 (for the twodimensional case) and Theorem 6 (for the three-dimensional case) specialize the results to these cases.

In the sequel, $\sim$ in expressions like $A \sim B$ means that both quantities can be uniformly bounded by constant multiples of each other. Likewise $\lesssim$ indicates inequalities up to constant factors.

\section{Wavelet Approximation with Local Refinement in One Dimension}

In this section we develop the one-dimensional means in order to approximate singular functions that are powers of distance functions to points. Instead of using explicit representations like (1), we derive an estimate in terms of weighted Sobolev norms. For this, we define a hierarchy of finite-dimensional spaces $V_{L}^{\beta} \subset V_{L+1}^{\beta} \subset H^{1}$ (where $\beta$ is a parameter indicating the local refinement toward the singularity) and corresponding 
projectors $P_{L}^{\beta}$, and prove that

$$
\operatorname{dim}\left(V_{L}^{\beta}\right) \leq C(p) 2^{L} \quad \text { and } \quad\left\|u-P_{L}^{\beta} u\right\|_{H^{1}([0,1])} \leq C(p) L^{1 / 2} 2^{-p L}\|u\|_{*},
$$

for functions $u$ with $\|u\|_{*}<\infty$ ( $p$ is the local polynomial degree of the ansatz functions). The norm $\|\cdot\|_{*}$ is a weighted Sobolev norm (and will be defined precisely in the sequel). The singularity functions of interest are in these weighted spaces.

The one-dimensional framework will later be used to infer $d$-dimensional results by sparse "tensor products" of the univariate results. In this regard, the use of hierarchic spaces is essential to our approach.

We begin by describing the wavelets we use. Then we define the locally (toward the singularity) refined projectors and ansatz spaces, as well as the weighted spaces. The last part of this section is devoted to the proof of the approximation property in terms of these weighted norms.

Let $\psi, \tilde{\psi}$ be compactly supported biorthogonal spline wavelets of (a fixed) degree $p \geq 1$ on the interval $[0,1]$, such that for

$$
u=\sum_{j=0}^{\infty} \sum_{k=1}^{k_{j}} c_{j k} \psi_{j k}=\sum_{j=0}^{\infty} \sum_{k=1}^{k_{j}}\left\langle u, \tilde{\psi}_{j k}\right\rangle_{L^{2}} \psi_{j k}
$$

the following norm equivalences (resp., estimates) hold:

$$
\begin{aligned}
& c_{1}\|u\|_{H^{t}([0,1])}^{2} \leq \sum_{j, k} 2^{2 t j} c_{j k}^{2} \leq c_{2}\|u\|_{H^{t}([0,1])}^{2} \quad \text { for } 0 \leq t<p+\frac{1}{2}, \\
& \sum_{\substack{j, k \\
j \leq L}} 2^{2 t j} c_{j k}^{2} \leq c_{2}\|u\|_{H^{t}([0,1])}^{2} \quad \text { for } p+\frac{1}{2} \leq t<p, \\
& \sum_{\substack{j, k \\
j \leq L}} 2^{2 t j} c_{j k}^{2} \leq c_{2} L\|u\|_{H^{t}([0,1])}^{2} \quad \text { for } t=p,
\end{aligned}
$$

where the constants $c_{1}$ and $c_{2}$ depend on $t$. Such wavelets are constructed, for instance, in [11]; see also [10]. For the one-sided norm estimates, see [24].

On each level $j$, the number of wavelets $\psi_{j k}$ is bounded by $p\left(2^{j}+N\right)$ with some global constant $N \in \mathbf{N}$. To simplify notation, we take $N=0$. We ignore the dependence of the indices $k$ on $p$, thus regarding an index $(j, k)$ standing for $p$ local wavelets basis functions. The support of a wavelet $\psi_{j k}$ is of order $2^{-j}$. Note that the norm equivalences are a consequence of vanishing moment properties of the wavelets (resp., duals).

We define now one-dimensional wavelet projectors $P_{L}^{\beta}$, which are designed to approximate singular functions of type $x \mapsto x^{\lambda}$. For this, let $I=\{(j, k) \in \mathbf{N} \times \mathbf{N}: j \in$ $\left.\mathbf{N}, 1 \leq k \leq 2^{j}\right\}$, and define for $L \in \mathbf{N}$ and fixed $\beta \in[0,1)$,

$$
\Lambda_{L}^{\beta} \equiv\left\{(j, k) \in I:\left(k 2^{-j}\right)^{\beta} \leq 2^{L-j}\right\} .
$$

The index set $\Lambda_{L}^{\beta}$ contains all index pairs $(j, k)$ with $j \leq L$, but considerably more toward the left endpoint 0 . But still it holds that

$$
\# \Lambda_{L}^{\beta} \lesssim p 2^{L},
$$


with a constant depending only on $\beta$. In the vicinity of 0 , the minimal support of wavelets indexed by $\Lambda_{L}^{\beta}$ is of order $2^{-L /(1-\beta)}$.

Definition 1 (Projector $P_{L}^{\beta}$ ). Let $u$ be a function with wavelet decomposition $u=\sum_{j, k}$ $c_{j k} \psi_{j k}$. Then the projector $P_{L}^{\beta}$ is defined by

$$
P_{L}^{\beta} u \equiv \sum_{(j, k) \in \Lambda_{L}^{\beta}} c_{j k} \psi_{j k}
$$

We denote by $V_{L}^{\beta}$ the image of $P_{L}^{\beta}$, that is, $V_{L}^{\beta}=\operatorname{span}\left\{\psi_{j k}:(j, k) \in \Lambda_{L}^{\beta}\right\} \subset H^{p}([0,1])$.

The following figure depicts a typical space $V_{L}^{\beta}$ : The basis functions spanning $V_{L}^{\beta}$ are marked by black dots. In this example, $L=2$ and $\beta=0.6$; the maximal occurring level in the vicinity of the origin is $L /(1-\beta)=5$.

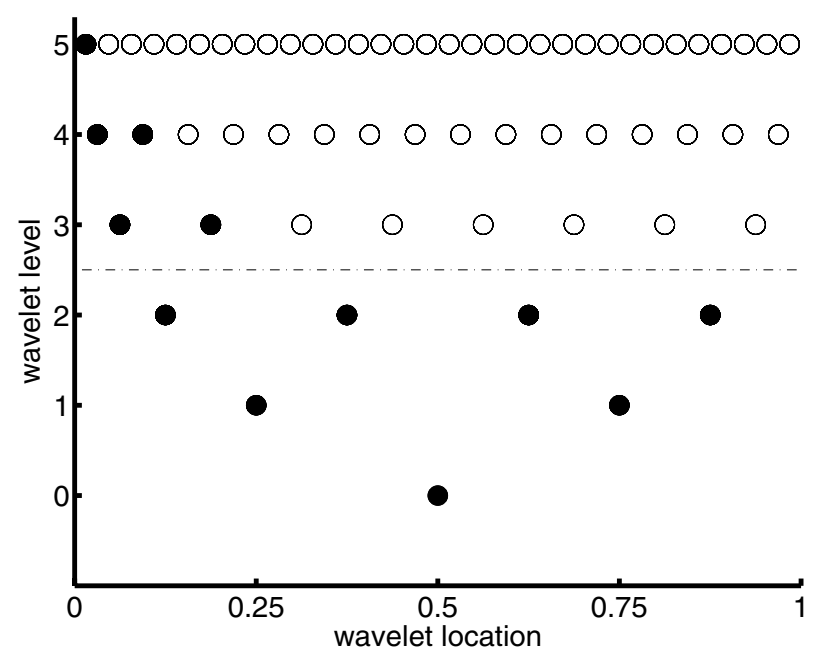

The projectors $P_{L}^{\beta}$ are simultaneously stable with respect to the $H^{t}$ norms, $0 \leq t<$ $p+\frac{1}{2}$.

Lemma 1 (Stability of $\left.P_{L}^{\beta}\right)$. Let $t \in\left[0, p+\frac{1}{2}\right)$. Then $P_{L}^{\beta}: H^{t}([0,1]) \rightarrow H^{t}([0,1])$ is bounded independently of $L$.

Proof. Using (3), we have

$$
\begin{aligned}
\left\|P_{L}^{\beta} u\right\|_{H^{t}([0,1])}^{2} & =\left\|\sum_{(j, k) \in \Lambda_{L}^{\beta}} c_{j k} \psi_{j k}\right\|_{H^{t}([0,1])}^{2} \sim \sum_{(j, k) \in \Lambda_{L}^{\beta}} 2^{2 t j} c_{j k}^{2} \\
& \leq \sum_{j, k} 2^{2 t j} c_{j k}^{2} \sim\|u\|_{H^{t}([0,1])}^{2} .
\end{aligned}
$$


Next, we will prove an approximation result for $P_{L}^{\beta}$ involving weighted Sobolev norms. For this, we first introduce these weighted spaces:

Definition 2 (Weighted spaces, $d=1$ ). For $m \in \mathbf{N}$, let $H_{\gamma}^{m}([0,1])$ be the space of all measurable functions $u$ such that

$$
\|u\|_{H_{\gamma}^{m}([0,1])}:=\left(\sum_{i=0}^{m}\left\|\left(D^{i} u\right) x^{\gamma}\right\|_{L^{2}([0,1])}^{2}\right)^{1 / 2}
$$

is finite. The expression $\|\cdot\|_{H_{\gamma}^{m}([0,1])}$ is a norm on this space.

We have the following embedding result:

Lemma 2 (Embedding, $d=1$ ). If $\gamma>p+\frac{1}{2}$, then the following embedding is continuous:

$$
H_{\gamma}^{p+1}([0,1]) \hookrightarrow L^{q}([0,1]) \quad \text { for } \quad q<\frac{1}{\gamma-\left(p+\frac{1}{2}\right)} .
$$

Proof. We first note that there exists a continuous extension operator $E: H_{\gamma}^{p+1}([0,1])$ $\rightarrow H_{\gamma}^{p+1}([0,2])$ with the property supp $E u \subset[0,2)$. This is due to the fact that close to the right boundary $\{1\}$ the space $H_{\gamma}^{p+1}$ coincides (in a local sense) with the space $H^{p+1}$.

Let now $\gamma>p+\frac{1}{2}$. We write

$$
\begin{aligned}
|u(x)|= & |E u(x)| \leq \int_{x}^{2}\left|(E u)^{\prime}(s)\right| d s \leq \int_{x}^{2} \int_{s_{1}}^{2} \cdots \int_{s_{p}}^{2}\left|D^{p+1}(E u)(t)\right| d t d s_{p} \cdots d s_{1} \\
= & \int_{x}^{2} \int_{s_{1}}^{2} \cdots \int_{s_{p}}^{2}\left|D^{p+1}(E u)(t) t^{\gamma}\right| t^{-\gamma} d t d s_{p} \cdots d s_{1} \\
\leq & \int_{x}^{2} \int_{s_{1}}^{2} \cdots \int_{s_{p-1}}^{2} \underbrace{\left(\int_{s_{p}}^{2}\left|D^{p+1}(E u)(t) t^{\gamma}\right|^{2} d t\right)^{1 / 2}} \\
& \times\left(\int_{s_{p}}^{2} t^{-2 \gamma} d t\right)^{1 / 2} d s_{p} \cdots D^{p+1}(E u) t^{\gamma} \|_{L^{2}([0,2))} d s_{1} \\
\lesssim & \|u\|_{H_{\gamma}^{p+1}([0,1])} \cdot \int_{x}^{2} \int_{s_{1}}^{2} \cdots \int_{s_{p-1}}^{2}\left(\int_{s_{p}}^{2} t^{-2 \gamma} d t\right)^{1 / 2} d s_{p} \cdots d s_{1} \\
\lesssim & \|u\|_{H_{\gamma}^{p+1}([0,1])} \cdot \int_{x}^{2} \int_{s_{1}}^{2} \ldots \int_{s_{p-1}}^{2} s_{p}^{1 / 2-\gamma} d s_{p} \cdots d s_{1} \\
\lesssim & \|u\|_{H_{\gamma}^{p+1}([0,1])}^{2} \cdot \int_{x}^{2} \int_{s_{1}}^{2} \ldots \int_{s_{p-2}}^{2} s_{p-1}^{3 / 2-\gamma} d s_{p-1} \cdots d s_{1} \\
\lesssim & \lesssim x^{p+1 / 2-\gamma}\|u\|_{H_{\gamma}^{p+1}([0,1])} \cdot \\
\vdots &
\end{aligned}
$$


Taking this to the power $q$, integrating over $[0,1]$, and taking to the power $1 / q$ yields

$$
\|u\|_{L^{q}([0,1])} \lesssim\|u\|_{H_{\gamma}^{p+1}([0,1])} \underbrace{\left(\int_{0}^{1} x^{q(p+1 / 2-\gamma)} d x\right)^{1 / q}}_{=: C} .
$$

$C$ is finite, if $q<1 /\left(\gamma-\left(p+\frac{1}{2}\right)\right)$.

Now we are able to prove the one-dimensional approximation result with respect to a weighted norm of the above type.

Lemma 3 (Weighted Norm Consistency of $P_{L}^{\beta}, d=1$ ). For

$$
1>\beta>\gamma>\frac{p+\frac{1}{2}}{p+1}
$$

and $u \in H_{\gamma(p+1)}^{p+1}([0,1])$ it holds that

$$
\left\|u-P_{L}^{\beta} u\right\|_{L^{2}([0,1])} \lesssim L^{1 / 2} 2^{-(p+1) L}\|u\|_{H_{\gamma(p+1)}^{p+1}([0,1])} .
$$

For

$$
\beta>\gamma>\frac{p-\frac{1}{2}}{p}
$$

and $u \in H_{\gamma p}^{p+1}([0,1])$ it holds that

$$
\left\|u-P_{L}^{\beta} u\right\|_{H^{1}([0,1])} \lesssim L^{1 / 2} 2^{-p L}\|u\|_{H_{\gamma p}^{p+1}([0,1])} .
$$

Proof. It suffices to prove the statements above for $u \in H^{p+1}([0,1])$. The lemma then follows from a density argument.

We split the interval $[0,1]$ into subintervals of length $2^{-L /(1-\beta)}$, for simplicity assuming $L /(1-\beta)$ to be an integer (otherwise rounded off). For $s=1, \ldots, 2^{L /(1-\beta)}$, set

$$
I(s)=\left[(s-1) 2^{-L /(1-\beta)}, s 2^{-L /(1-\beta)}\right] .
$$

Let $j_{0}(s)$ be a bound for the minimal local level of wavelets $\psi_{j k}$ with $(j, k) \notin \Lambda_{L}^{\beta}$ and supp $\psi_{j k} \cap I(s) \neq \varnothing$. Examining the condition $\left(s 2^{-L /(1-\beta)}\right)^{\beta} \leq 2^{L-j}$, we see that $j_{0}(s)$ can be chosen to be

$$
j_{0}(s)=\frac{L}{1-\beta}-\beta \log _{2} s .
$$

Applying (3), we estimate

$$
\left\|u-P_{L}^{\beta} u\right\|_{L^{2}(I(s))}^{2} \lesssim \sum_{\substack{(j, k) \notin \Lambda_{L}^{\beta} \\ \text { supp } \psi_{j k} \cap I(s) \neq \varnothing}} c_{j k}^{2}
$$




$$
\begin{aligned}
& =\sum_{j \geq j_{0}(s)} 2^{-2(p+1) j} \sum_{\substack{k:(j, k) \notin \Lambda_{L}^{\beta} \\
\operatorname{supp} \psi_{j k} \cap I(s) \neq \varnothing}} 2^{2(p+1) j} c_{j k}^{2} \\
& \lesssim \sum_{j \geq j_{0}(s)} j 2^{-2(p+1) j}\|u\|_{H^{p+1}(I(s))}^{2} \lesssim j_{0}(s) 2^{-2(p+1) j_{0}(s)}\|u\|_{H^{p+1}(I(s))}^{2} .
\end{aligned}
$$

Using $2^{-2(p+1) j_{0}(s)}=2^{-2(p+1) L}\left(s 2^{-L /(1-\beta)}\right)^{2(p+1) \beta}$ and $j_{0}(s) \lesssim L$, we infer

$$
\left\|u-P_{L}^{\beta} u\right\|_{L^{2}(I(s))}^{2} \lesssim L 2^{-2(p+1) L}\left\|u\left(s 2^{-L /(1-\beta)}\right)^{(p+1) \beta}\right\|_{H^{p+1}(I(s))}^{2} .
$$

Away from 0 , i.e., in the case of $s>1$, we have $s 2^{-L /(1-\beta)} \sim x$ for $x \in I(s)$. This yields

$$
\left\|u-P_{L}^{\beta} u\right\|_{L^{2}\left(\left[2^{-L /(1-\beta)}, 1\right]\right)}^{2} \lesssim L 2^{-2(p+1) L}\left\|u x^{(p+1) \beta}\right\|_{H^{p+1}\left(\left[2^{-L /(1-\beta)}, 1\right]\right)}^{2} .
$$

In the case of $s=1$, we employ $L^{2}$ stability of the projectors $P_{L}^{\beta}$ (Lemma 1 ) and the embedding result, Lemma 2.

If $\gamma>\left(p+\frac{1}{2}\right) /(p+1)$, i.e., $\gamma(p+1)>p+\frac{1}{2}$, we estimate

$$
\left\|u-P_{L}^{\beta} u\right\|_{L^{2}(I(1))}^{2} \lesssim\|u\|_{L^{2}(I(1))}^{2} \leq|I(1)|^{1-2 / q}\|u\|_{L^{q}(I(1))}^{2} \lesssim|I(1)|^{1-2 / q}\|u\|_{H_{\gamma(p+1)}^{p+1}([0,1])}^{2} .
$$

Here, $q<1 /\left(\gamma(p+1)-\left(p+\frac{1}{2}\right)\right)$ corresponding to Lemma 2. To guarantee $|I(1)|^{1-2 / q} \leq$ $2^{-2(p+1) L}$, we get the constraint $q \geq 1 /\left(\beta(p+1)-\left(p+\frac{1}{2}\right)\right)$. Hence, if $\beta>\gamma$, we can choose such a $q$. Together with (9), the $L^{2}$ estimate follows.

The $H^{1}$ estimate is done in an analogous fashion. Using (3), we get

$$
\begin{aligned}
\left\|u-P_{L}^{\beta} u\right\|_{H^{1}(I(s))}^{2} & \lesssim \sum_{\substack{(j, k) \notin \Lambda_{L}^{\beta} \\
\operatorname{supp} \psi_{j k} \cap I(s) \neq \varnothing}} 2^{2 j} c_{j k}^{2} \\
& =\sum_{j \geq j_{0}(s)} 2^{-2 p j} \sum_{\substack{k:(j, k) \notin \Lambda_{L}^{\beta} \\
\operatorname{supp} \psi_{j k} \cap I(s) \neq \varnothing}} 2^{2(p+1) j} c_{j k}^{2} \\
& \lesssim \sum_{j \geq j_{0}(s)} j 2^{-2 p j}\|u\|_{H^{p+1}(I(s))}^{2} \lesssim j_{0}(s) 2^{-2 p j_{0}(s)}\|u\|_{H^{p+1}(I(s))}^{2} .
\end{aligned}
$$

Using $2^{-2 p j_{0}(s)}=2^{-2 p L}\left(s 2^{-L /(1-\beta)}\right)^{2 p \beta}$ and $j_{0}(s) \lesssim L$, we infer

$$
\left\|u-P_{L}^{\beta} u\right\|_{H^{1}(I(s))}^{2} \lesssim L 2^{-2 p L}\left\|u\left(s 2^{-L /(1-\beta)}\right)^{p \beta}\right\|_{H^{p+1}(I(s))}^{2} .
$$

Away from 0 , i.e., in the case of $s>1$, we have $s 2^{-L /(1-\beta)} \sim x$ for $x \in I(s)$. This yields

$$
\left\|u-P_{L}^{\beta} u\right\|_{H^{1}\left(\left[2^{-L /(1-\beta)}, 1\right]\right)}^{2} \lesssim L 2^{-2 p L}\left\|u x^{p \beta}\right\|_{H^{p+1}\left(\left[2^{-L /(1-\beta)}, 1\right]\right)}^{2} .
$$

In the case $s=1$, we estimate, using Lemma 2,

$$
\begin{aligned}
\left\|D\left(u-P_{L}^{\beta} u\right)\right\|_{L^{2}(I(1))}^{2} & \lesssim\|D u\|_{L^{2}(I(1))}^{2} \leq|I(1)|^{1-2 / q}\|D u\|_{L^{q}(I(1))}^{2} \\
& \lesssim|I(1)|^{1-2 / q}\|u\|_{H_{\gamma p}^{p+1}(I(1))}^{2},
\end{aligned}
$$

if $q<1 /\left(\gamma p-\left(p-\frac{1}{2}\right)\right)$ and $\gamma p>p-\frac{1}{2}$. To guarantee $|I(1)|^{1-2 / q} \leq 2^{-2 p L}$, we get the constraint $q \geq 1 /\left(\beta p-\left(p-\frac{1}{2}\right)\right)$. Hence, if $\beta>\gamma$, we can choose such a $q$. Together with (10), the $H^{1}$ estimate (8) follows. 
Remark 1. The function $u: x \mapsto x^{\lambda}$ belongs to $H_{\gamma p}^{p+1}([0,1])$, if

$$
\gamma>1-\frac{2 \lambda-1}{2 p} \text {. }
$$

Thus, if $\lambda>\frac{1}{2}$, there is such a $\gamma<1$.

Remark 2. In [4] a similar one-dimensional result for Chui prewavelet bases of degree 2 and 3 was proven, using a slightly different refinement toward the origin and a different method of proof.

A similiar approach was already undertaken in [23].

\section{Wavelet Approximation with Local Refinement in Two Dimensions}

We come now to the two-dimensional case. Consider the unit square $[0,1]^{2} \subset \mathbf{R}^{2}$. We define a wavelet basis by taking tensor products of the univariate wavelets introduced above, i.e., the set $\left\{\psi_{j k} \otimes \psi_{j^{\prime} k^{\prime}}:(j, k) \in I,\left(j^{\prime}, k^{\prime}\right) \in I\right\}$. Note the anisotropy of the supports of these wavelets.

We will employ a sparse tensor product rather than a full tensor product of the projectors $P_{L}^{\beta}$. The sparse tensor product projector has comparable approximation properties to the full one, but uses a remarkably lower-dimensional subspace to project onto. We will give the details in the following.

Let $\beta \in[0,1)$ be fixed and let $L \in \mathbf{N}$. Then we define the index sets

$$
\hat{\Lambda}_{L}^{\beta} \equiv \bigcup_{i+j \leq L} \Lambda_{i}^{\beta} \times \Lambda_{j}^{\beta}
$$

and the trial spaces

$$
\hat{V}_{L}^{\beta}:=\operatorname{span}\left\{\psi_{j k} \otimes \psi_{j^{\prime} k^{\prime}}:\left(j, k, j^{\prime}, k^{\prime}\right) \in \hat{\Lambda}_{L}^{\beta}\right\}=\bigoplus_{i+j \leq L} V_{i}^{\beta} \otimes V_{j}^{\beta} .
$$

From (5), we see

$$
\# \hat{\Lambda}_{L}^{\beta}=\operatorname{dim}\left(\hat{V}_{L}^{\beta}\right) \lesssim p^{2} L 2^{L} .
$$

Definition 3 (Projector $\hat{P}_{L}^{\beta}$ ). Let $u$ be a function with wavelet decomposition

$$
u=\sum_{j, k, j^{\prime}, k^{\prime}} c_{j k j^{\prime} k^{\prime}} \psi_{j k} \otimes \psi_{j^{\prime} k^{\prime}}
$$

Then the projector $\hat{P}_{L}^{\beta}$ is defined by

$$
\hat{P}_{L}^{\beta} u \equiv \sum_{j, k, j^{\prime}, k^{\prime} \in \hat{\Lambda}_{L}^{\beta}} c_{j k j^{\prime} k^{\prime}} \psi_{j k} \otimes \psi_{j^{\prime} k^{\prime}}
$$

Definition 4. Let $(s, t) \in \mathbf{N}^{2}$. Then we denote by

$$
H^{s, t}\left([0,1]^{2}\right)
$$


the space of all measurable functions $u:[0,1]^{2} \rightarrow \mathbf{R}$, such that the norm

$$
\|u\|_{H^{s, t}\left([0,1]^{2}\right)}:=\left(\sum_{\substack{0 \leq i \leq s \\ 0 \leq j \leq t}}\left\|D_{1}^{i} D_{2}^{j} u\right\|_{L^{2}\left([0,1]^{2}\right)}^{2}\right)^{1 / 2}
$$

is finite. That is, $H^{s, t}\left([0,1]^{2}\right)=H^{s}([0,1]) \otimes H^{t}([0,1])$.

Lemma 4. The projectors $\hat{P}_{L}^{\beta}$ are stable in $H^{1} \otimes L^{2}$ independently of $L$.

Proof. From the one-dimensional norm equivalences, one readily derives norm equivalences for tensor product spaces in the following form:

$$
\left\|\sum_{j, k, j^{\prime}, k^{\prime}} c_{j k j^{\prime} k^{\prime}} \psi_{j k} \otimes \psi_{j^{\prime} k^{\prime}}\right\|_{H^{s, t}\left([0,1]^{2}\right)}^{2} \sim \sum_{j, k, j^{\prime}, k^{\prime}} 2^{2 s j+2 t j^{\prime}} c_{j k j^{\prime} k^{\prime}}^{2}, \quad 0 \leq s, t<p+\frac{1}{2} .
$$

For $s=1$ and $t=0$, one accordingly has, for $u=\sum c_{j k j^{\prime} k^{\prime}} \psi_{j k} \otimes \psi_{j^{\prime} k^{\prime}}$,

$$
\begin{aligned}
\left\|\hat{P}_{L}^{\beta} u\right\|_{H^{1,0}\left([0,1]^{2}\right)}^{2} & =\left\|\sum_{\left(j, k, j^{\prime}, k^{\prime}\right) \in \hat{\Lambda}_{L}^{\beta}} c_{j k j^{\prime} k^{\prime}} \psi_{j k} \otimes \psi_{j^{\prime} k^{\prime}}\right\|_{H^{1,0}\left([0,1]^{2}\right)}^{2} \sim \sum_{\left(j, k, j^{\prime}, k^{\prime}\right) \in \hat{\Lambda}_{L}^{\beta}} 2^{2 j} c_{j k j^{\prime} k^{\prime}}^{2} \\
& \leq \sum_{j, k, j^{\prime}, k^{\prime}} 2^{2 j} c_{j k j^{\prime} k^{\prime}}^{2} \sim\|u\|_{H^{1,0}\left([0,1]^{2}\right)}^{2}
\end{aligned}
$$

Definition 5. For $\sigma, \tau \geq 0$ and $p, q \in \mathbf{N}$ let

$$
H_{\sigma, \tau}^{p, q}\left([0,1]^{2}\right):=H_{\sigma}^{p}([0,1]) \otimes H_{\tau}^{q}([0,1]) .
$$

We will derive a consistency estimate for $\hat{P}_{L}^{\beta}$ by an abstract tensor product argument following the lines of [25]. Since $\|\varphi\|_{H^{1}\left([0,1]^{2}\right)}^{2} \leq\|\varphi\|_{H^{1,0}\left([0,1]^{2}\right)}^{2}+\|\varphi\|_{H^{0,1}\left([0,1]^{2}\right)}^{2}$, it suffices (by symmetry) to estimate the $H^{1,0}\left([0,1]^{2}\right)$ norm of the error $u-\hat{P}_{L}^{\beta} u$. To simplify notation, we set

$$
\|\cdot\|:=\|\cdot\|_{H^{1}([0,1])} \otimes\|\cdot\|_{L^{2}([0,1])}=\|\cdot\|_{H^{1,0}\left([0,1]^{2}\right)} .
$$

Let $Q_{1, i}^{\beta}$ be the orthogonal projection onto $V_{i}^{\beta}$ with respect to the $H^{1}([0,1])$ norm, and let $Q_{2, i}^{\beta}$ be the orthogonal projection onto $V_{i}^{\beta}$ with respect to the $L^{2}([0,1])$ norm.

We set

$$
\hat{Q}_{L}^{\beta}:=\sum_{i+j \leq L}\left(Q_{1, i}^{\beta}-Q_{1, i-1}^{\beta}\right) \otimes\left(Q_{2, j}^{\beta}-Q_{2, j-1}^{\beta}\right)
$$

$\hat{Q}_{L}^{\beta}$ is the orthogonal projection onto $\hat{V}_{L}^{\beta}$ with respect to $\|\cdot\|=\|\cdot\|_{H^{1,0}\left([0,1]^{2}\right)}$. Note that, in general, $\hat{Q}_{L}^{\beta} \neq \hat{P}_{L}^{\beta}$ (but, of course, image $\hat{Q}_{L}^{\beta}=$ image $\hat{P}_{L}^{\beta}=\hat{V}_{L}^{\beta}$ ). 
By stability of $\hat{P}_{L}^{\beta}$ in $H^{1} \otimes L^{2}$, Lemma 4 , we have

$$
\begin{aligned}
\left\|u-\hat{P}_{L}^{\beta} u\right\| & =\left\|u-\hat{Q}_{L}^{\beta} u+\hat{Q}_{L}^{\beta} u-\hat{P}_{L}^{\beta} u\right\|=\left\|u-\hat{Q}_{L}^{\beta} u+\hat{P}_{L}^{\beta} \hat{Q}_{L}^{\beta} u-\hat{P}_{L}^{\beta} u\right\| \\
& =\left\|\left(\operatorname{id}-\hat{P}_{L}^{\beta}\right)\left(u-\hat{Q}_{L}^{\beta} u\right)\right\| \leq c\left\|u-\hat{Q}_{L}^{\beta} u\right\| .
\end{aligned}
$$

It therefore suffices to estimate the error $\left\|u-\hat{Q}_{L}^{\beta} u\right\|$ to get an approximation result for $\hat{P}_{L}^{\beta}$.

By orthogonality of the projectors $\hat{Q}_{i}^{\beta}$ we have, quite generally,

$$
\begin{aligned}
\left\|u-\hat{Q}_{L}^{\beta} u\right\|^{2}= & \left\|u-\sum_{i+j \leq L}\left(Q_{1, i}^{\beta}-Q_{1, i-1}^{\beta}\right) \otimes\left(Q_{2, j}^{\beta}-Q_{2, j-1}^{\beta}\right) u\right\|^{2} \\
= & \left\|\sum_{i+j \geq L+1}\left(Q_{1, i}^{\beta}-Q_{1, i-1}^{\beta}\right) \otimes\left(Q_{2, j}^{\beta}-Q_{2, j-1}^{\beta}\right) u\right\|^{2} \\
= & \left\|\sum_{i=0}^{L+1}\left(Q_{1, i}^{\beta}-Q_{1, i-1}^{\beta}\right) \otimes\left(\mathrm{id}-\hat{Q}_{2, L-i}^{\beta}\right) u\right\|^{2} \\
& +\left\|\sum_{i=L+2}^{\infty}\left(Q_{1, i}^{\beta}-Q_{1, i-1}^{\beta}\right) \otimes \mathrm{id} u\right\|^{2} \\
= & \sum_{i=0}^{L+1}\left\|\left(Q_{1, i}^{\beta}-Q_{1, i-1}^{\beta}\right) \otimes\left(\mathrm{id}-\hat{Q}_{2, L-i}^{\beta}\right) u\right\|^{2}+\left\|\left(\mathrm{id}-Q_{1, L+1}^{\beta}\right) \otimes \mathrm{id} u\right\|^{2} .
\end{aligned}
$$

Applying once more orthogonality, we estimate the last line by

$$
\leq \sum_{i=0}^{L+1}\left\|\left(\mathrm{id}-Q_{1, i-1}^{\beta}\right) \otimes\left(\mathrm{id}-\hat{Q}_{2, L-i}^{\beta}\right) u\right\|^{2}+\left\|\left(\mathrm{id}-Q_{1, L+1}^{\beta}\right) \otimes \mathrm{id} u\right\|^{2} .
$$

By stability of the orthogonal projections $Q_{r, i}^{\beta}$, we can translate this estimate back into an estimate involving the projectors $P_{L}^{\beta}$. To see this (using simplified notation), let $Q, P, A$ be projectors with $Q$ stable. Then it holds that

$$
\begin{aligned}
\|(\mathrm{id}-Q) \otimes A u\| & =\|(\mathrm{id}-P) \otimes A u+P \otimes A u-Q \otimes A u\| \\
& =\|(\mathrm{id}-P) \otimes A u+Q P \otimes A u-Q \otimes A u\| \\
& =\|(\mathrm{id}-P) \otimes A u+(Q \otimes \mathrm{id})(P \otimes A u-\mathrm{id} \otimes A) u\| \\
& =\|(\mathrm{id} \otimes \mathrm{id}-Q \otimes \mathrm{id})((\mathrm{id}-P) \otimes A) u\| \leq c\|(\mathrm{id}-P) \otimes A u\| .
\end{aligned}
$$

Hence, we finally get

$$
\left\|u-\hat{P}_{L}^{\beta} u\right\|^{2} \leq c\left(\sum_{i=0}^{L+1}\left\|\left(\mathrm{id}-P_{i-1}^{\beta}\right) \otimes\left(\mathrm{id}-P_{L-i}^{\beta}\right) u\right\|^{2}+\left\|\left(\mathrm{id}-P_{L+1}^{\beta}\right) \otimes \mathrm{id} u\right\|^{2}\right) .
$$


Now using the one-dimensional consistency result, Lemma 3, we infer

$$
\begin{aligned}
\left\|u-\hat{P}_{L}^{\beta} u\right\|^{2} \lesssim & \sum_{i=0}^{L+1} L^{2} 2^{-2 p(i-1)} 2^{-2(p+1)(L-i)}\|u\|_{H_{\gamma p, \gamma(p+1)}^{p+1, p+1}\left([0,1]^{2}\right)}^{2} \\
& +L 2^{-2 p(L+1)}\|u\|_{H_{\gamma p}^{p+1}([0,1]) \otimes L^{2}([0,1])}^{2},
\end{aligned}
$$

hence,

$$
\left\|u-\hat{P}_{L}^{\beta} u\right\|^{2} \lesssim L^{2} 2^{-2 p L}\|u\|_{H_{\gamma p, \gamma(p+1)}^{p+1, p+1}\left([0,1]^{2}\right)}^{2}
$$

We summarize:

Theorem 1. For

$$
1>\beta>\gamma>\frac{p+\frac{1}{2}}{p+1}
$$

and $u \in H_{\gamma p, \gamma(p+1)}^{p+1, p+1}\left([0,1]^{2}\right) \cap H_{\gamma(p+1), \gamma p}^{p+1, p+1}\left([0,1]^{2}\right)$, it holds that

$$
\left\|u-\hat{P}_{L}^{\beta} u\right\|_{H^{1}\left([0,1]^{2}\right)} \lesssim L 2^{-p L}\left(\|u\|_{H_{\gamma p, \gamma(p+1)}^{p+1, p+1}\left([0,1]^{2}\right)}+\|u\|_{H_{\gamma(p+1), \gamma p}^{p+1, p+1}\left([0,1]^{2}\right)}\right) .
$$

Remark 3. Note, that it trivially holds that

$$
H^{2(p+1)}\left([0,1]^{2}\right) \subset H_{\gamma p, \gamma(p+1)}^{p+1, p+1}\left([0,1]^{2}\right) \cap H_{\gamma(p+1), \gamma p}^{p+1, p+1}\left([0,1]^{2}\right) \quad \text { for } \quad \gamma \geq 0 .
$$

In an analogous fashion, one proves

Theorem 2. For

$$
1>\beta>\gamma>\frac{p+\frac{1}{2}}{p+1}
$$

and $u \in H_{\gamma(p+1), \gamma(p+1)}^{p+1, p+1}\left([0,1]^{2}\right)$, it holds that

$$
\left\|u-\hat{P}_{L}^{\beta} u\right\|_{L^{2}\left([0,1]^{2}\right)} \lesssim L^{3 / 2} 2^{-(p+1) L}\|u\|_{H_{\gamma(p+1), \gamma(p+1)}^{p+1, p+1}\left([0,1]^{2}\right)} .
$$

Remark 4. In [21], P. Oswald has considered best $N$ term approximation from sparse grid spaces built on the univariate Haar system $(p=0)$. He derives approximation rates in Sobolev spaces $H^{s}\left([0,1]^{2}\right),-1<s<\frac{1}{2}$. In the case of approximation in $L^{2}([0,1])$, i.e., $s=0$, he achieves an approximation rate of $N^{-1}(\log N)^{3 / 2}$ for singularity functions of type $(1 ; \alpha, \alpha), \alpha<\frac{1}{2}$. Here, a function $f\left(x_{1}, x_{2}\right)$ is said to be of type $(m ; \alpha, \alpha)$, if $\left|\partial_{1}^{k} \partial_{2}^{l} f\right| \leq C x_{1}^{-(\alpha+k)} x_{2}^{-(\alpha+l)}$ for $0 \leq k, l \leq m$.

A singular function of type $(1 ; \alpha, \alpha)$ belongs to $H_{\gamma, \gamma}^{1,1}\left([0,1]^{2}\right)$ if $\gamma>\frac{1}{2}+\alpha$. Hence, our result is consistent with Oswald's. 


\subsection{Application to Elliptic Singularities in Two Dimensions}

Consider an elliptic boundary value problem on a polygonal domain $\Omega \subset \mathbf{R}^{2}$ :

$$
L u=f \quad \text { in } \Omega, \quad B u=g \quad \text { on } \partial \Omega,
$$

where $L$ is a smooth, uniformly elliptic, linear second-order differential operator in divergence form, $B$ is a smooth boundary operator of Dirichlet or Neumann type, and $f$ and $g$ are smooth functions.

The weak solution $u$ is smooth (in the Sobolev scale) in the interior of $\Omega$, but exhibits corner singularities, which reduce global regularity dramatically. In general, $u \notin H^{2}(\Omega)$. It is well-known, see [5], [6], [7], that there holds a decomposition

$$
u=u_{\mathrm{reg}}+u_{\mathrm{sing}},
$$

with (arbitrarily) smooth $u_{\text {reg }}$, which we specialize to $u_{\text {reg }} \in H^{2(p+1)}(\Omega)$, and (depending on the chosen smoothness of $u_{\text {reg }}$ ) a finite sum

$$
u_{\text {sing }}=\sum^{<\infty} \operatorname{Re}\left(r_{i}^{\lambda_{i}}\left(\log r_{i}\right)^{\mu_{i}} \Phi_{i}\left(\varphi_{i}\right) \eta_{i}(x)\right),
$$

(in local polar coordinates $\left(r_{i}, \varphi_{i}\right)$ associated to the corners) with smooth cut-off functions $\eta_{i}$, smooth functions $\Phi_{i}$ of the angle coordinates $\varphi_{i}$, and parameters $\lambda_{i} \in \mathbf{C}, \mu_{i} \in \mathbf{N}_{0}$.

For Dirichlet-Dirichlet corners, one knows $\operatorname{Re} \lambda_{i}>\frac{1}{2}$, and for Dirichlet-Neumann corners, $\operatorname{Re} \lambda_{i}>\frac{1}{4}$.

We prove now that this type of singularity can be approximated at a rate $p$ in the $H^{1}$ norm. Note that we can allow $\operatorname{Re} \lambda>0$.

Theorem 3. Let

$$
u(x)=\operatorname{Re}\left(|x|^{\lambda}(\log |x|)^{\mu} \Phi(\varphi) \eta(x)\right)+g(x) \in H^{1}\left([0,1]^{2}\right)
$$

with smooth $\eta$ and $\Phi$, parameters $\lambda \in \mathbf{C}, \operatorname{Re}(\lambda)>0, \mu \in \mathbf{N}_{0}$, and a function $g \in$ $H^{2(p+1)}\left([0,1]^{2}\right)$. Then there are $\beta, \gamma$ (depending on $\left.\lambda\right)$ with

$$
1>\beta>\gamma>\frac{p+\frac{1}{2}}{p+1}
$$

such that

$$
u \in H_{\gamma p, \gamma(p+1)}^{p+1, p+1}\left([0,1]^{2}\right) \cap H_{\gamma(p+1), \gamma p}^{p+1, p+1}\left([0,1]^{2}\right) .
$$

This means there is a constant $C$ depending via $\beta$ and $\gamma$ only on $\lambda$, such that

$$
\left\|u-\hat{P}_{L}^{\beta} u\right\|_{H^{1}\left([0,1]^{2}\right)} \leq C L 2^{-p L}\left(\|u\|_{H_{\gamma, \gamma, \gamma(p+1)}^{p+1, p+1}\left([0,1]^{2}\right)}+\|u\|_{H_{\gamma(p+1), \gamma p}^{p+1, p+1}\left([0,1]^{2}\right)}\right) .
$$

Concerning approximability in $L^{2}$, we have:

If $\operatorname{Re}(\lambda)>-1$ and the remaining assumptions as above are met, then there are $\beta, \gamma$ with

$$
1>\beta>\gamma>\frac{p+\frac{1}{2}}{p+1}
$$


such that

$$
u \in H_{\gamma(p+1), \gamma(p+1)}^{p+1, p+1}\left([0,1]^{2}\right) .
$$

This means there is a constant $C$ such that

$$
\left\|u-\hat{P}_{L}^{\beta} u\right\|_{L^{2}\left([0,1]^{2}\right)} \leq C L^{3 / 2} 2^{-(p+1) L}\|u\|_{H_{\gamma(p+1), \gamma(p+1)}^{p+1, p+1}\left([0,1]^{2}\right)} .
$$

Remark 5. Note that we are only concerned with the approximation of certain singular terms, which are defined on the unit square. In this paper, we do not address the question of handling complex geometries with sparse grids within a numerical scheme.

Proof. The norm of $H_{\gamma p, \gamma(p+1)}^{p+1, p+1}\left([0,1]^{2}\right)$ is given by

$$
\|u\|_{H_{\gamma p, \gamma(p+1)}^{p+1, p+1}\left([0,1]^{2}\right)}=\left(\sum_{0 \leq i, j \leq p+1} \int_{[0,1]^{2}}\left|\left(D_{1}^{i} D_{2}^{j} u\right) x_{1}^{\gamma p} x_{2}^{\gamma(p+1)}\right|^{2} d x\right)^{1 / 2} .
$$

Estimating $x_{1}^{\gamma p} x_{2}^{\gamma(p+1)}$ by $|x|^{\gamma(2 p+1)}$, we see that $\operatorname{Re}\left(|x|^{\lambda}(\log |x|)^{\mu}\right) \in H_{\gamma p, \gamma(p+1)}^{p+1, p+1}$ $\left([0,1]^{2}\right)$ if

$$
\gamma>1-\frac{\lambda}{2 p+1}
$$

The same holds for the space $H_{\gamma(p+1), \gamma p}^{p+1, p+1}\left([0,1]^{2}\right)$.

The smooth factors $\eta$ and $\Phi$ leave the regularity class unchanged, and $g$ is trivially in the space $H_{\gamma p, \gamma(p+1)}^{p+1, p+1}\left([0,1]^{2}\right) \cap H_{\gamma(p+1), \gamma p}^{p+1, p+1}\left([0,1]^{2}\right)$, see Remark 3 .

Hence, if $\lambda>0$, we can find $1>\beta>\gamma>1-\lambda /(2 p+1)$ such that the assumptions of Theorem 1 are met.

The proof of the $L^{2}$ estimate is done analogously.

\section{Generalization to $d$ Dimensions}

The $d$-dimensional case with $d>2$ follows from the two-dimensional one by iteration. We consider the domain $[0,1]^{d}$ and the tensor product basis

$$
\left\{\psi_{j_{1} k_{1} \cdots j_{d} k_{d}}=\psi_{j_{1} k_{1}} \otimes \cdots \otimes \psi_{j_{d} k_{d}}:\left(j_{i}, k_{i}\right) \in I, i=1, \ldots, d\right\} .
$$

Let $\beta \in[0,1)$ be fixed and let $L \in \mathbf{N}$. Then we define the index sets

$$
\hat{\Lambda}_{L, d}^{\beta} \equiv \bigcup_{j_{1}+\cdots+j_{d} \leq L} \Lambda_{j_{1}}^{\beta} \times \cdots \times \Lambda_{j_{d}}^{\beta}
$$

and the trial spaces

$$
\hat{V}_{L, d}^{\beta}:=\operatorname{span}\left\{\psi_{j_{1} k_{1} \cdots j_{d} k_{d}}:\left(j_{1}, k_{1}, \ldots, j_{d}, k_{d}\right) \in \hat{\Lambda}_{L, d}^{\beta}\right\}=\bigoplus_{j_{1}+\cdots+j_{d} \leq L} V_{j_{1}}^{\beta} \otimes \cdots \otimes V_{j_{d}}^{\beta} .
$$

From (5) we see that

$$
\# \hat{\Lambda}_{L, d}^{\beta}=\operatorname{dim}\left(\hat{V}_{L, d}^{\beta}\right) \lesssim p^{d} L^{d-1} 2^{L} .
$$

The constant depends here, and in the sequel, additionally on the dimension $d$. 
Definition 6 (Projector $\hat{P}_{L, d}^{\beta}$ ). Let $u$ be a function with wavelet decomposition

$$
u=\sum_{j_{1}, k_{1}, \ldots, j_{d}, k_{d}} c_{j_{1} k_{1} \cdots j_{d} k_{d}} \psi_{j_{1} k_{1}} \otimes \cdots \otimes \psi_{j_{d} k_{d}} .
$$

Then the projector $\hat{P}_{L, d}^{\beta}$ is defined by

$$
\hat{P}_{L, d}^{\beta} u \equiv \sum_{j_{1}, k_{1}, \ldots, j_{d}, k_{d} \in \hat{\Lambda}_{L, d}^{\beta}} c_{j_{1} k_{1} \cdots j_{d} k_{d}} \psi_{j_{1} k_{1}} \otimes \cdots \otimes \psi_{j_{d} k_{d}}
$$

Definition 7. Let $\left(s_{1}, \ldots, s_{d}\right) \in \mathbf{N}^{d}$. Then we denote by

$$
H^{s_{1}, \ldots, s_{d}}\left([0,1]^{d}\right)
$$

the space of all measurable functions $u:[0,1]^{d} \rightarrow \mathbf{R}$, such that the norm

$$
\|u\|_{H^{s_{1}, \ldots, s_{d}\left([0,1]^{d}\right)}}:=\left(\sum_{0 \leq j_{i} \leq s_{i}}\left\|D_{1}^{j_{1}} \cdots D_{d}^{j_{d}} u\right\|_{L^{2}\left[[0,1]^{d}\right)}^{2}\right)^{1 / 2}
$$

is finite. That is, $H^{s_{1}, \ldots, s_{d}}\left([0,1]^{d}\right)=\bigotimes_{i=1, \ldots, d} H^{s_{i}}([0,1])$.

Definition 8. For $\sigma_{1}, \ldots, \sigma_{d} \geq 0$ and $p_{1}, \ldots, p_{d} \in \mathbf{N}$ let

$$
H_{\sigma_{1}, \ldots, \sigma_{d}}^{p_{1}, \ldots, p_{d}}\left([0,1]^{d}\right):=\bigotimes_{i=1, \ldots, d} H_{\sigma_{i}}^{p_{i}}([0,1]) .
$$

We further introduce the abbreviation

$$
H(\gamma, p, d):=\bigcap_{k=1, \ldots, d}\left(\bigotimes_{i=1, \ldots, d} H_{\gamma\left(p+1-\delta_{k i}\right)}^{p+1}([0,1])\right)
$$

and take as a norm the summed up norms.

Remark 6. Note, that it trivially holds that

$$
H^{d(p+1)}\left([0,1]^{d}\right) \subset H(\gamma, p, d) \quad \text { for } \quad \gamma \geq 0 .
$$

With the latter notation, Theorem 1 may be rephrased as follows:

$\beta>\gamma>\frac{p+\frac{1}{2}}{p+1}, \quad u \in H(\gamma, p, d) \quad \Rightarrow \quad\left\|u-\hat{P}_{L, 2}^{\beta} u\right\|_{H^{1}\left([0,1]^{d}\right)} \lesssim L 2^{-p L}\|u\|_{H(\gamma, p, d)}$.

Iterating the argument from Section 3, we infer the following two theorems: 
Theorem $4\left(H^{1}\right.$ consistency of $\left.\hat{P}_{L}^{\beta}\right)$. For

$$
1>\beta>\gamma>\frac{p+\frac{1}{2}}{p+1}
$$

and $u \in H(\gamma, p, d)$, it holds that

$$
\left\|u-\hat{P}_{L, d}^{\beta} u\right\|_{H^{1}\left([0,1]^{d}\right)} \lesssim L^{d / 2} 2^{-p L}\|u\|_{H(\gamma, p, d)} .
$$

Letting $N_{L}:=\operatorname{dim} \hat{V}_{L}^{\beta} \lesssim p^{d} L^{d-1} 2^{L}$ be the number of degrees of freedom, this means that

$$
\begin{aligned}
\left\|u-\hat{P}_{L, d}^{\beta} u\right\|_{H^{1}\left([0,1]^{d}\right)} & \lesssim N_{L}^{-p}\left(\log _{2} N_{L}\right)^{s}\|u\|_{H(\gamma, p, d)}, \\
s & =(d-1) p+d / 2 .
\end{aligned}
$$

Theorem $5\left(L^{2}\right.$ consistency of $\left.\hat{P}_{L}^{\beta}\right)$. For

$$
1>\beta>\gamma>\frac{p+\frac{1}{2}}{p+1}
$$

and $u \in H_{\gamma(p+1), \ldots, \gamma(p+1)}^{p+1, \ldots, p}\left([0,1]^{d}\right)$, it holds that

$$
\left\|u-\hat{P}_{L, d}^{\beta} u\right\|_{L^{2}\left([0,1]^{d}\right)} \lesssim L^{d-1 / 2} 2^{-(p+1) L}\|u\|_{H_{\gamma(p+1) \ldots, \gamma(p+1)}^{p+1, \ldots, p+1}\left([0,1]^{d}\right)} .
$$

In terms of numbers of degrees of freedom, this means that

$$
\begin{aligned}
\left\|u-\hat{P}_{L, d}^{\beta} u\right\|_{L^{2}\left([0,1]^{d}\right)} & \lesssim N_{L}^{-(p+1)}\left(\log _{2} N_{L}\right)^{s}\|u\|_{H_{\gamma(p+1) \ldots, \gamma(p+1)}^{p+1, \ldots, 1}\left([0,1]^{d}\right)}, \\
s & =(d-1) p+2 d-\frac{3}{2} .
\end{aligned}
$$

Remark 7. There holds the continuous inclusion

$$
H(\gamma, p, d) \subset H_{\gamma(p+1), \ldots, \gamma(p+1)}^{p+1, \ldots, p+1}\left([0,1]^{d}\right) .
$$

Remark 8. By interpolating the estimates of Theorems 4 and 5, consistency estimates in $H^{s}\left([0,1]^{d}\right), 0<s<1$ are readily obtained.

Remark 9. The function $x \mapsto \operatorname{Re}|x|^{\lambda}$ on $[0,1]^{d}$ belongs to $H(\gamma, p, d)$, if

$$
\gamma>1-\frac{(d-2)+2 \lambda}{2 d(p+1)-2}
$$

So, if

$$
\lambda>1-d / 2
$$

we can find such a $\gamma<1$. 
More generally, let $\rho$ be the distance to a face of $[0,1]^{d}$ of dimension $k$ containing the origin (for $k=0$ the corner 0 , for $k=1$ an edge containing 0 , etc.). Then the function $x \mapsto \operatorname{Re} \rho^{\lambda}$ on $[0,1]^{d}$ belongs to some $H(\gamma, p, d)$ with $\gamma<1$, if

$$
\lambda>1-(d-k) / 2 .
$$

Note that this condition on the exponent $\lambda$ coincides with the condition of membership in $H^{1}\left([0,1]^{d}\right)$, which indicates the sharpness of our result with respect to powers of distance functions to lower-dimensional faces.

\subsection{Application to Elliptic Singularities in Three Dimensions}

We apply our approximation result to elliptic corner-edge singularities in three dimensions. Consider on a polyhedral domain $\Omega$ (for instance, $[0,1]^{3}$ ) the boundary value problem

$$
L u=f \quad \text { in } \Omega, \quad B u=g \quad \text { on } \partial \Omega,
$$

where $L$ is a smooth, uniformly elliptic, linear second-order differential operator in divergence form, $B$ is a smooth boundary operator of face-wise Dirichlet or Neumann type, and $f$ and $g$ are smooth functions.

As in the two-dimensional case, the weak solution $u$ is smooth away from corners and edges, but exhibits singularities toward the singularities of the domain. The solution's asymptotics toward corners and edges can be described by weighted Sobolev spaces, and we quote only one result (in simplified form), which was proven by Maz'ya and Roßmann (Theorem 2 in [19]):

There is $\varepsilon>0$ (depending on the domain), such that for $\delta \in \mathbf{R}$ and $\ell \in \mathbf{N}$ with $|\delta-\ell-1|<\varepsilon$ there holds

$$
f \in V_{\delta}^{\ell} \Rightarrow u \in V_{\delta}^{\ell+2} .
$$

Here, the space $V_{\delta}^{\ell}$ is defined as the set of functions $v$, for which

$$
\left(\int_{\Omega}\left(\prod r_{i}^{2 \delta}\right)\left(\prod\left(\rho_{j} / r\right)^{2 \delta}\right) \sum_{|\alpha| \leq \ell} \rho^{2(|\alpha|-\ell)}\left|D^{\alpha} u\right|^{2} d x\right)^{1 / 2}
$$

is finite. The functions $r_{i}$ are the distance functions to the vertices of $\Omega$, the functions $\rho_{j}$ are the distance funtions to the edges of $\Omega$, and $r=\min _{i} r_{i}, \rho=\min _{j} \rho_{j}$.

For the sake of simplicity of exposition, we specialize this to the case of one "active" cube-like corner (which is supposed to be the origin). Then the norm of $V_{\delta}^{\ell}$ reads

$$
\left(\int_{\Omega} r^{2 \delta}\left(\prod_{j=1}^{3}\left(\rho_{j} / r\right)^{2 \delta}\right) \sum_{|\alpha| \leq \ell} \rho^{2(|\alpha|-\ell)}\left|D^{\alpha} u\right|^{2} d x\right)^{1 / 2} .
$$

Using the general relations $\rho \leq \rho_{j} \leq r$, a calculation shows that

$$
f \in C^{\infty}(\bar{\Omega}) \Rightarrow f \in V_{\delta}^{\ell} \quad \text { for all } \quad \delta \geq \ell .
$$


Now choose $\ell=3 p+1$ and $\delta=\ell+1-\varepsilon_{0}=3 p+2-\varepsilon_{0}$ for some $0<\varepsilon_{0}<$ $\min \{\varepsilon, 1\}$. From the above shift theorem, we see that $u \in V_{\delta}^{3 p+3}$. But this readily implies $u \in H(\gamma, p, 3)$ for

$$
\frac{\delta}{3 p+2}=\frac{3 p+2-\varepsilon_{0}}{3 p+2}<\gamma<1 \text {. }
$$

We conclude:

Theorem 6. The solution $u$ of problem (15) lies locally in some space $H(\gamma, p, 3)$ with $\gamma<1$ and may therefore (as a function transferred to the unit cube) be approximated at a rate $p$ in the $H^{1}$ norm.

Acknowledgments. Member of the IHP network "Breaking Complexity" of the EC, contract No. HPRN-CT-2002-00286, and supported by the Swiss Federal Office for Science and Education under grant BBW02.0418. The author would like thank Professor Christoph Schwab for inducting him into this subject and for many helpful discussions.

\section{References}

1. TH. APEL (1999): Anisotropic Finite Elements: Local Estimates and Applications. Advances in Numerical Mathematics. Stuttgart: Teubner.

2. A. Barinka, T. Barsch, P. Charton, A. Cohen, S. Dahlke, W. Dahmen, K. Urban (2001): Adaptive wavelet schemes for elliptic problems-Implementations and numerical experiments. SIAM J. Sci. Comput., 23:910-939.

3. P. BINEV, W. DAHMEN, R. DEVORE (2002): Adaptive finite element methods with convergence rates. Preprint.

4. C. BourgeoIS, S. NiCAISE (1999): Approximation par préondelettes augmentée de l'équation de la plaque libre polygonale. C. R. Acad. Sci. Paris Sér. I, 329:727-730.

5. M. Bourlard, M. Dauge, M. Lubuma, S. NiCAise (1990): Coefficients des singularitétes pour des problèmes aux limites elliptiques sur un domaine à points coniques. I: Résultats généraux pour le probème de Dirichlet. RAIRO Modél. Anal. Numér., 24:27-52.

6. M. Bourlard, M. DAuge, M. Lubuma, S. NiCAISE (1990): Coefficients des singularitétes pour des problèmes aux limites elliptiques sur un domaine à points coniques. II: Quelques opératuers particuliers. RAIRO Modél. Anal. Numér., 24:343-367.

7. M. Bourlard, M. Dauge, M. Lubuma, S. NiCAise (1992): Coefficients of the singularities for elliptic boundary value problems on domains with conical points. III: Finite element methods on polygonal domains. SIAM J. Numer. Anal., 29:136-155.

8. H. Bungartz, M. Griebel (1999): A note on the complexity of solving Poisson's equation for spaces of bounded mixed derivatives. J. Complity, 15:167-199.

9. A. COHEN, W. DAHMEN, R. DEVORE (2000): Adaptive wavelet methods for elliptic operator equations: Convergence rates. Math. Comp., 70(233):27-75.

10. W. DAHMEN, A. KUNOTH, K. URBAN (1999): Biorthogonal spline-wavelets on the interval-Stability and moment conditions. Appl. Comput. Harmonic Anal., 6:132-196.

11. W. DAHMEN, R. SCHNEIDER (1998): Wavelets with complementary boundary conditions-Function spaces on the cube. Resultate Math., 34:255-293.

12. M. DAuge (1988): Elliptic Boundary Value Problems on Corner Domains: Smoothness and Asymptotics of Solutions. Berlin: Springer-Verlag.

13. R. DeVore (1998): Nonlinear approximation. In: Acta Numerica 7. Cambridge: Cambridge University Press, pp. 51-150. 
14. D. Gilbarg, N. S. TRUdinger (1998): Elliptic Partial Differential Equations of Second Order. New York: Springer-Verlag.

15. M. GRIEBEL, S. KNAPEK (2000): Optimized tensor-product approximation spaces. Constr. Approx., 16:525-540.

16. M. GRIEBEL, P. OSWALD, T. SCHIEKOFER (1999): Sparse grids for boundary integral equations. Numer. Math., 83:279-312.

17. P. GRISVARD (1985): Elliptic Problems in Nonsmooth Domains. Boston: Pitman.

18. V. A. Kozlov, V. G. MaZYA, J. Rossmann (1997): Elliptic Boundary Value Problems in Domains with Point Singularities. Providence, RI: American Mathematical Society.

19. V. B. MAZ'YA, J. ROßMANN (1991): On the Agmon-Miranda maximum principle. Ann. Global Anal. Geom., 9:253-303.

20. P. MORIN, R. NOCHETTO, K. SIEBERT (2000): Data oscillation and convergence of adaptive FEM. SIAM J. Numer. Anal., 38(2).

21. P. OSWALD (1999): On best n-term approximation by Haar functions in $H^{s}$-norms. In: Metric Function Theory and Related Topics in Analysis (Nicolskij, Kashin, Izaak, eds.). Moscow: AFC, pp. 137-163.

22. P. OSWALD (1999): Best n-term capacitance approximation on sparse grids. Proc. 12th Intern. Conf. on Domain Decomp. Methods in Science and Engineering (T. Chan et al., eds.). Chiba, pp. 437-444.

23. T. VON PETERSDORFF, CH. SCHWAB (1995): Boundary element methods with wavelets and mesh refinement. Research Report No. 95-10, Seminar für Angewandte Mathematik, ETH Zürich.

24. T. VON PETERSDORFF, CH. SCHWAB, R. SCHNEIDER (1997): Multiwavelets for second-kind integral equations. SIAM J. Numer. Anal., 34:2212-2227.

25. СH. SchwaB, R. A. TOdOR (2003): Sparse finite elements for elliptic problems with stochastic data. SAM ETHZ Report 2002-05 (available online under: http://www.sam.math.ethz.ch/reports/). To appear in Numer. Math.

26. CH. Zenger (1991): Sparse Grids; in Parallel Algorithms for PDE. Proc. 6th GAMM Seminar, Kiel (W. Hackbusch, ed.). Braunschweig: Vieweg, pp. 241-251.

P.-A. Nitsche

Seminar for Applied Mathematics

ETH-Zentrum

CH-8092 Zürich

Switzerland 